\title{
Three-dimensional pulmonary monitoring using focused electrical impedance measurements
}

\author{
Jakob Orschulik ${ }^{1,2}$, Diana Pokee ${ }^{1}$, Tobias Menden ${ }^{1}$, Steffen Leonhardt ${ }^{1}$, Marian Walter ${ }^{1}$ \\ 1. Medical Information Technology, Helmholtz Institute for Biomedical Engineering, RWTH Aachen University, Aachen, Germany \\ 2. E-mail any correspondence to: orschulik@hia.rwth-aachen.de
}

\begin{abstract}
Lung pathologies such as edema, atelectasis or pneumonia are potentially life threatening conditions. Especially in critically ill and mechanically ventilated patients, an early diagnosis and treatment is crucial to prevent an Acute Respiratory Distress Syndrome [1]. Thus, continuous monitoring tool for the lung condition available at the bedside would be highly appreciated. One concept for this is Electrical Impedance Tomography (EIT). In EIT, an electrode belt of typically 16 or 32 electrodes is attached at the body surface and multiple impedance measurements are performed. From this, the conductivity change inside the body is reconstructed in a two-dimensional image. In various studies, EIT proved to be a useful tool for quantifying recruitment maneuvers, the assessment of the ventilation homogeneity, the detection of lung edema or perfusion monitoring [2, 3, 4, 5]. Nevertheless, the main problem of EIT is the low spatial resolution (compared to CT) and the limitation to two dimensional images. In this paper, we try to address the latter issue: Instead of projecting conductivity changes onto a two-dimensional image, we adjust electrode positions to focus single tetrapolar measurements to specific, three-dimensional regions of interest. In earlier work, we defined guidelines to achieve this focusing [6] 7]. In this paper, we demonstrate in simulations and in a water tank experiment that applying these guidelines can help to detect pathologies in specific lung regions.
\end{abstract}

Keywords: Electrical Impedance Analysis, Pulmonary Monitoring, Regional Analysis

\section{Introduction}

The continuous monitoring of the air distribution inside the lungs is of great clinical interest at the bedside, especially in mechanically ventilated patients. One promising concept to achieve this is Electrical Impedance Tomography (EIT). The general idea is to reconstruct impedance changes inside the body from multiple impedance measurements at the body surface [5]. These measurements are typically performed using 16 or 32 electrodes, which are positioned on a belt around the thorax at a defined height, typically between the 4th and 6st intercostal space. A small, alternating current is injected into the body between two electrodes and the resulting voltage drop across the other electrodes is measured. Then, the injection position is shifted and the measurement is repeated, until all measurements at all injection positions have been recorded. This concept allows the reconstruction of images, which typically display the impedance change with respect to a time reference. The big advantages of EIT are the high temporal resolution, the bedside availability and its non-invasivity. Today, commercially available devices feature up to 50 frames per second.

The biggest drawback, however, is the poor spatial resolution. When using 16 electrodes, 104 linear independent measurements are recorded, from which an image with a resolution of typically $32 \times 32$ pixels is reconstructed. Thus, EIT is rather used for functional monitoring than for anatomical analysis. Furthermore, impedance changes from above and below the electrode plane are mapped onto the image plane. While this allows to detect, for example, dorsal or ventral ventilation inhomogeneities, no cranial or caudal information can be resolved from the measurement data.

The lack of three-dimensional information has been addressed by different groups in different applications 
[8, $9,10,11,12$. For ventilation monitoring, the most intuitive way is to add a second electrode plane allowing measurements at different heights and to reconstruct a three dimensional impedance change distribution. However, adding a second plane causes additional issues such as the determination of the optimal injection and measurement pattern and the optimal placement and distance of the electrode planes. Furthermore, the complexity of the reconstruction problem is increased. Recently, different concepts for three dimensional reconstruction have been developed. One way is to expand the popular, two-dimensional GREIT algorithm into the third dimension [13, 14]. Another concept investigates the addition of prior anatomical information by mapping the dual-belt-measurements on specific lung lobes resulting in a lobe-based image reconstruction [15]. Both concepts use dual belt EIT electrode configurations for their work.

In previous work, we demonstrated that impedance measurements can be focused on desired regions of interest inside the lung by adjusting electrode positions [6. 7]. The idea of this work is to utilize these optimized positions to perform a regional impedance analysis using only single tetrapolar measurements for each respective region of interest. We do not aim to reconstruct images. Instead, we seek to analyze temporal activity or spectroscopic information of desired lung regions of interest.

This paper is structured as follows: section 2 (Materials and Methods) gives an overview about the evaluation criteria and the simulation and measurement setup. In section 3 , the results of the simulation and the experiment are demonstrated. The discussion of the results and the conclusions are presented in the final section.

\section{Materials and Methods}

In this section, the materials and methods will be introduced. First, the concept of focused tetrapolar impedance measurements will be explained. Then, the simulation model and setup will be presented. Finally, the experimental setup with the water tank phantom will be introduced.

\section{Ethical approval}

The conducted research is not related to either human or animals use.

\section{Focusing of Tetrapolar Impedance Measurements}

In bioimpedance measurements, a tetrapolar electrode setup is typically used to measure an electrical impedance. In this setup, two electrodes are used to inject a small, alternating current into the body while two separate electrodes are measuring the resulting voltage drop across those electrodes. In contrast to impedance-based imaging techniques such as Electrical Impedance Tomography, where multiple measurements are performed at different electrode locations, single tetrapolar measurements typically do not contain any spatial information, especially if the electrodes are placed far away from each other, for example in hand-to-hand or hand-to-foot configurations. However, when optimizing the electrode positions to specific placement strategies, measurements can be focused to desired regions of interest [16]. In particular, we showed in earlier work that impedance measurements can be focused to specific lung regions [6, 7]. For better understanding and readability of this article, the criteria will be repeated in this section.

In general, the measured impedance of a tetrapolar impedance measurement can be described using eq. (1):

$$
\underline{Z}=\int_{v} \frac{1}{\sigma(v)} \cdot \vec{J}_{L E}(v) \cdot \vec{J}_{L I}(v) \mathrm{d} v,
$$

where $\underline{Z}$ is the measured impedance, $\vec{J}_{L E}(v)$ and $\vec{J}_{L I}(v)$ are the current density of the injecting and sensing electrode pair, respectively, and $\sigma(v)$ is the conductivity in a given location $v[17,18,19]$. It should be noted that $\underline{Z}$ is not the physical impedance of the body or between one electrode pair, but should be interpreted rather as a local transfer function given an input injected current and an output measured voltage drop. Thus, while analyzing $\underline{Z}$ does not necessarily allow an interpretation of the dielectric properties of the investigated body part, an analysis of the impact of certain locations on $\underline{Z}$ can be estimated.

In particular, we extract two measures from eq. (1): The sensitivity $S(v)$ and the local impedance contribution $\zeta(v)$ :

$$
\begin{aligned}
& S(v)=\vec{J}_{L E}(v) \cdot \vec{J}_{L I}(v), \\
& \zeta(v)=\frac{1}{\sigma(v)} \cdot \vec{J}_{L E}(v) \cdot \vec{J}_{L I}(v)=\frac{1}{\sigma(v)} \cdot S(v) .
\end{aligned}
$$

The sensitivity $S(v)$ describes the impact of a local conductivity change on the measured impedance, while the local impedance contribution $\zeta(v)$ describes the contribution of a specific location $v$ to the measured transfer impedance $\underline{Z}$. Both the sensitivity and the local impedance contribution can be both positive and negative. A local conductivity increase in areas with positive local sensitivity will result in a decrease of the measured impedance, whereas in areas with negative local sensitivity, a local increase in conductivity will increased 
the measured impedance. For local impedance contribution, the behavior is similar: areas with a positive local impedance contribution increase the measured impedance, while areas with a negative contribution decrease the measured transfer impedance.

Now, in order to evaluate a tetrapolar electrode configuration with respect to its capability to focus on a specific region of interest (ROI) inside the lung, criteria were defined which are beneficial for focused impedance measurement [6, 7]: Let $\mathrm{L}_{\mathrm{ROI}}$ be the ROI inside the lung. Let further $\mathrm{L}_{\overline{\mathrm{ROI}}}$ be the remainder of the lung. Then, the following criteria can be defined:

1. A high Selectivity:

$$
\text { Sel }=\frac{\left|\mu\left(S_{\mathrm{L}_{\mathrm{ROI}}}\right)\right|}{\mu\left(\left|S_{\mathrm{L}_{\overline{\mathrm{ROI}}}}\right|\right)} .
$$

The selectivity measures the mean sensitivity inside the ROI compared to the mean sensitivity in the remainder of the lung. Thus, it is a measure on how much a conductivity change inside the ROI will be visible in the measured impedance compared to a sensitivity change in the remainder of the lung. The higher the sensitivity, the higher the impact of an impedance change inside ROI on the measurement data.

\section{A high Homogeneity:}

$$
H o m=\left|\frac{\mu\left(S_{\mathrm{L}_{\mathrm{ROI}}}\right)}{s t d\left(S_{\mathrm{L}_{\mathrm{ROI}}}\right)}\right| .
$$

The homogeneity measures the distribution of the sensitivity inside the ROI. A high homogeneity value means that changes in the complete ROI have a similar impact on the measured impedance.

3. A high Impedance Contribution Ratio:

$$
I_{\%}=\left|\frac{\sum \zeta_{\mathrm{L}_{\mathrm{ROI}}}}{\sum \zeta}\right| \cdot 100 \% .
$$

The Impedance Contribution Ratio shows, how much the ROI contributes to the measured impedance. The higher $I \%$, the higher the impact of the ROI on the actual impedance value. If for example $I_{\%}=50 \%$, the ROI accounts for half of the measured impedance value. However, $I \%$ does not measure the impact of an impedance change in this ROI.

\section{A high Absolute Impedance Contribution Ratio:}

$$
I_{a b s}=\frac{\sum\left|\zeta_{\mathrm{L}_{\mathrm{ROI}}}\right|}{\sum|\zeta|} \cdot 100 \%
$$

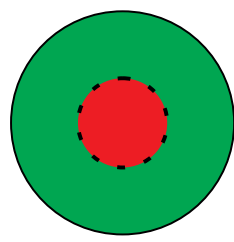

a.)

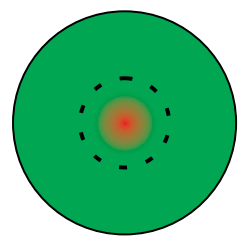

b.)

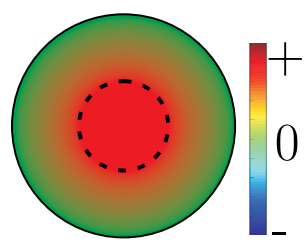

C.)
Fig. 1: Sample sensitivity distributions and their impact on the criteria Selectivity Sel and Homogeneity Hom. a.) shows the perfect sensitivity distribution with infinitely high Selectivity and Homogeneity values. b.) has a high Selectivity, but a low Homogeneity as areas close to the edge of the ROI have no sensitivity. In contrast, c.) has a high Homogeneity, but also a low Selectivity as lots of sensitivity is present in regions outside the ROI. Note, that the local impedance contribution can be determined by multiplying the sensitivity with the reciprocal local conductivity. Figure adapted from [7].

In contrast to the normal Impedance Contribution Ratio in eq. (6), the Absolute Impedance

Contribution Ratio measures the contribution of the $\mathrm{ROI}$ on the entire impedance activity, where activity means that both positive and negative local impedance contribution are taken into account.

Fig. 1 shows sample sensitivity distributions and their impact on the criteria. Here, the ROI is highlighted with the dashed circle in the center. Fig. 1 a.) shows the ideal distribution, where a high sensitivity is present inside the $\mathrm{ROI}$ while the sensitivity is zero outside the ROI. This ideal case, however, cannot be achieved by single tetrapolar measurements, as the sensitivity will not be zero everywhere outside the ROI, especially if the electrodes are placed outside the ROI, as the current density is highest close to the electrodes. Fig. 1 b.) shows a distribution, where a high sensitivity peak is visible inside the ROI. This distribution possesses a high Selectivity, but a low Homogeneity value. In Fig. 1 c.), the sensitivity is spread homogeneously inside the ROI and drops slowly outside the ROI. In this distribution, the Homogeneity value is high while the Selectivity is low. The local impedance contribution can be determined by multiplying the Sensitivity and the local conductivity. Thus, while only areas with a nonzero sensitivity contribute to the measured impedance, also areas with a low sensitivity may have a significant contribution to the measured impedance, if they occur in areas with a low local conductivity.

In this paper, we use the results obtained in [6] as a basis, where guidelines for electrode placement were established in a simulation study. We use these guidelines in order to perform simulations in Matlab and a water tank experiment. 


\section{Simulation Setup}

In order to avoid results that are only valid in a specific model, the simulation setup in this study is different to the simulation setup used for our previous optimization of the electrode configurations [6, 7]. While in the previous study CST has been used for simulation, the simulations in this study were performed in Matlab (MathWorks Inc., USA) using the EIDORS framework [20] and NETGEN [21]. Furthermore, a female model has been used in the previous study while the geometry of a male human thorax from [14] was used in this study. Only the derived electrode positions were applied to this study leading to the assumption that the results shown in this paper are not limited to the finite element model or simulation environment.

The lung was split into eight regions of interest (right/left, back/front, bottom/top) and for each ROI, a specific tetrapolar electrode setup was placed at the body surface based on the aforementioned guidelines. Fig. 2 shows the model, including the lung and the heart, and the tetrapolar configuration for the left, back bottom and the right, front, top region of interest. The respective $\mathrm{ROI}$ is highlighted in yellow. The values for the complex, frequency-dependent conductivity of the different organs were set according to the Gabriel database [22]. The background was set to the conductivity value of muscle tissue. Breathing was simulated by varying the conductivity of the lung between the conductivity value of deflated lung $(0 \%$ inflated) and the conductivity value for inflated lung (100\% inflated). For one breathing cycle, 21 breathing levels were simulated. Fig. 3 demonstrates the breathing activity for one cycle.

Three different pathologies were simulated in this study: atelectasis, pneumonia and edema [23]. In atelectasis, parts of the lungs are collapsed. Thus, in order to simulate an atelectasis, the ROI of the lung which suffers from atelectasis was set to $0 \%$ inflated while the other lung parts performed a normal breathing step as described above. In pneumonia, typically, parts of the lungs become inflamed. Thus, an combination of blood and pus is accumulated in the affected regions. Pus is characterized by the high amount of cells. As no conductivity value for pus is available in the Gabriel database, the conductivity of the affected regions was set to the conductivity value of the spleen, which has similar properties regarding cell structure, in order to simulate pneumonia. In edema, body fluid is accumulated inside the affected lung regions. Hence, the respective lung regions were simulated to be filled with isotonic saline solution to simulate lung edema. All simulations were performed at each of the 21 respiration levels. Additionally, in order to provide both a temporal and a spectroscopic analysis, simulations of the complex, frequency-dependent impedance measurements from $10 \mathrm{kHz}$ to $1 \mathrm{MHz}$ were also performed at each of the 21 lung states.

\section{Water tank phantom}

In order to validate the focusing capability of the electrode configurations, an experimental measurement using a water tank phantom was performed. The tank consists of four individual lung lobes, which are represented by balloons. Fig. 4 shows an image of the water tank.

The tank is filled with saline solution. Each lung lobe can be activated and deactivated manually by adjusting the connecting valve. Impedance measurements were performed using the Draeger EEK2 EIT device. To compare the experimental results to the simulation, both conventional 16 electrode single belt EIT measurements and impedance measurements focused on the regions left bottom and right top were performed. After a $30 \mathrm{~s}$ baseline measurement, each lung lobe was separately disabled for $30 \mathrm{~s}$.

\section{Results}

In this section, the results of the simulations and experiments, as introduced in the Materials and Methods, will be presented. Additionally, a comparison to simulated EIT images will be shown.

\section{Simulation Results}

The simulations results in this chapter will be presented for two exemplary regions of interest: The region left, back, bottom (lbb) and the region right, front, top ( $\mathrm{rtt}$ ). This is motivated by different clinical scenarios: Typically, the back regions are affected by atelectasis, especially in mechanically ventilated patients when lying on the back. One way of addressing this issue is to turn the patient around, which might lead to atelectasis in the then dependent front region. Furthermore, front top regions are typically associated with pneumothorax, where no ventilation activity will be present as an accumulation of air inside the pleural cave prevents the ventilation in this regions.

The evaluation criteria introduced in the Materials and Methods section were applied to the respective region of interest. The numerical results for both regions are shown in Tab. 1. 


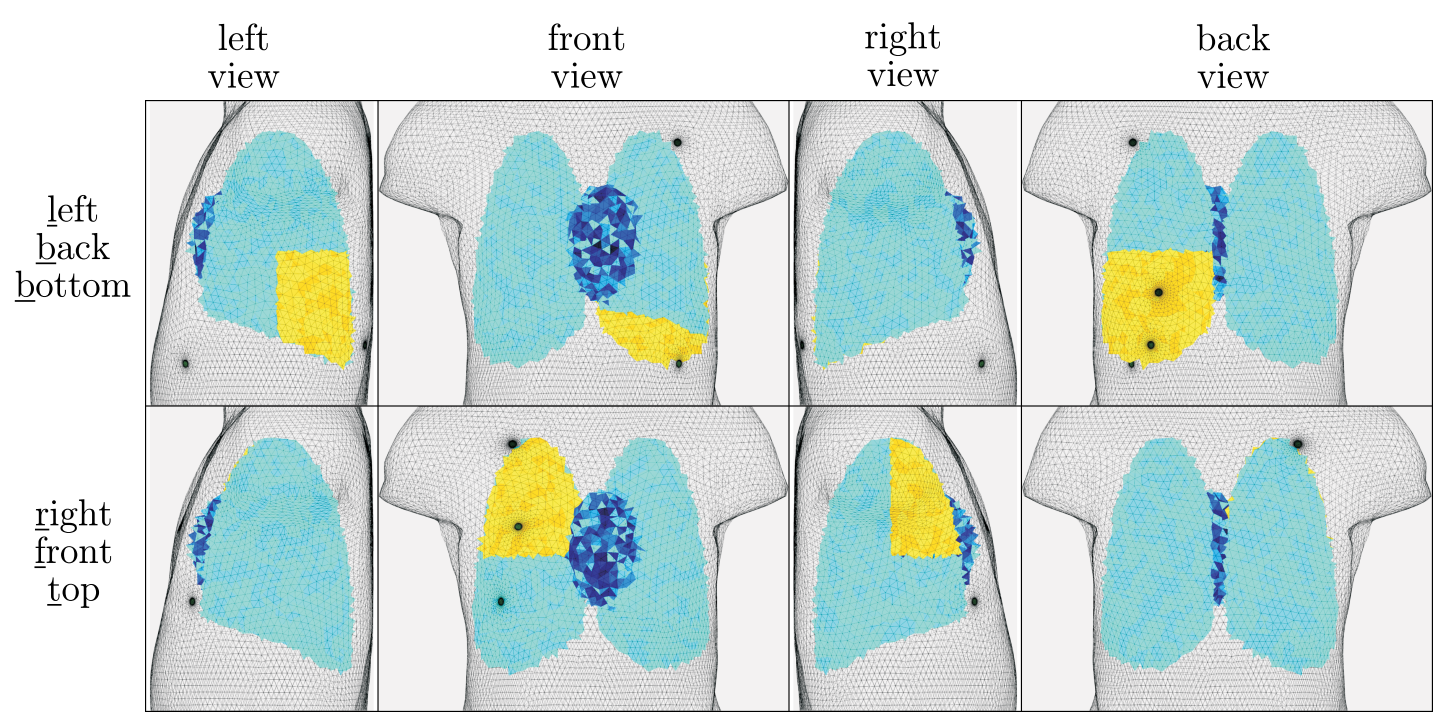

Fig. 2: Finite element model used in this study (model from [14]). The lungs were split into eight regions of interest. For each ROI, a specific tetrapolar electrode configuration was placed at the body surface. In this paper, the results for the regions of interest left, back, bottom and right, front, top region will be presented. The respective electrode configurations are based on the results in [6] and shown from different views in the two rows. The focused ROI is highlighted in yellow.

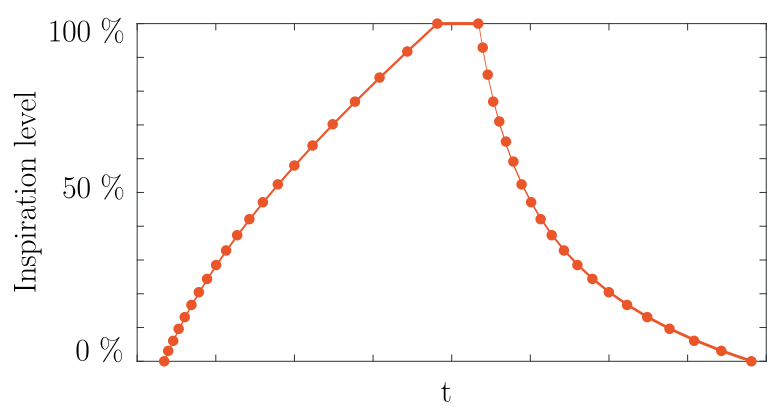

Fig. 3: Breathing cycle for the simulation of ventilation. The conductivity of the lungs was simulated on 21 levels between fully deflated and fully inflated.

\begin{tabular}{|c|c|c|c|c|}
\hline Region & Sel & Hom & $I_{\%}$ & $I_{a b s}$ \\
\hline $\mathrm{lbb}$ & 31.3 & 0.56 & $2.94 \%$ & $0.11 \%$ \\
\hline $\mathrm{rft}$ & 9.15 & 1.18 & $7.44 \%$ & $0.07 \%$ \\
\hline
\end{tabular}

Tab. 1: Numerical results of the evaluation for the regions left, back, bottom (lbb) and right, front, top ( $\mathrm{rft})$. The respective electrode positions are shown in Fig. 2

In both regions, the selectivity is greater than one, which means that conductivity changes inside the ROI have a higher impact on the measured impedance than conductivity changes elsewhere. Furthermore, the contribution of the ROI in the measured impedance is approx. $3 \%$ in the $\mathrm{lbb}$ region and approx. $7.5 \%$ in the $\mathrm{rt}$ region. While this may not seem much, one should keep in mind that other parts of the thorax contribute to the measured impedance itself. Typically, however, these parts, such as the muscles or bones, do not change their conductivity during a breathing cycle so that the selectivity is the most important criterion to detect local

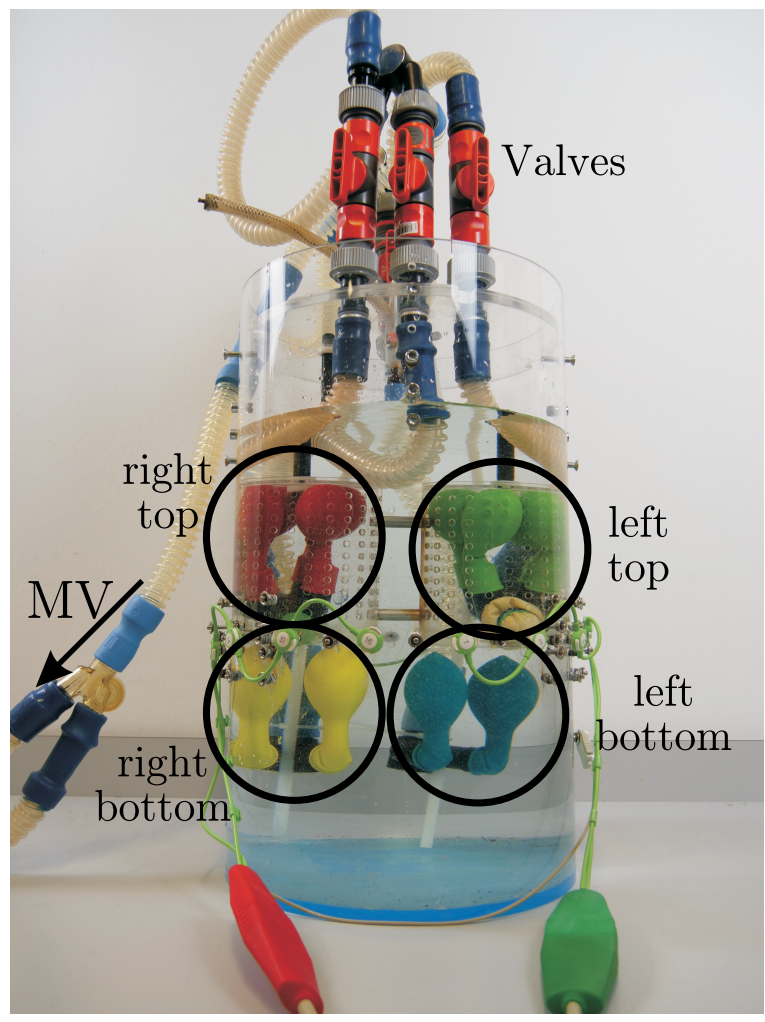

Fig. 4: Water tank phantom used for experimental validation. Four lung lobes are represented by balloons. All lungs can be opened and closed separately by an individual valve. The ventilation is realized by a mechanical ventilator (MV). The fourth valve is not visible as it is behind the other valves. In this image, the measurement setup for the EIT reference measurement using a single electrode belt is visualized. 
conductivity changes.

In Fig. 5a a simulation result for an impedance measurement focused on the ROI lbb during one breathing cycle is shown. The measurements were simulated at a frequency of $10 \mathrm{kHz}$ and the absolute value of the complex impedance is presented. In blue, the healthy state where all lung regions are ventilated is shown. In red, an atelectasis is simulated inside the ROI. It is apparent, that the impedance curve is highly affected by the lack of ventilation inside the region of interest. Furthermore, in green, an atelectasis is simulated in the left front bottom (Ifb) region of the lung, which is adjacent to the $\mathrm{lbb}$ focus region. During this simulation, almost no impact on the measurement result can be observed. This corresponds to the high selectivity of the electrode configuration of 31.3 for the lbb region. The impedance change inside the ROI has a much higher impact on the measurement result than an impedance change in other lung regions.

In Fig. 5b, the impact of the different pathologies on the measurement during one breathing cycle is presented. It is apparent that the impedance change during the breathing cycle decreases compared to the impedance change in healthy state. This is not surprising, as the affected lung region does not participate in the ventilation activity. It can be also observed that the baseline of the measurement is dependent on the type of pathology. This pathology dependent impedance is visualized in Fig. 5c. where the complex impedance spectrum in the frequency range between $10 \mathrm{kHz}$ and $1 \mathrm{MHz}$ is shown. It is apparent that each pathology has a different impact on the measured spectrum. Especially when comparing the healthy state to the pneumonia, it is visible that a pathological lobe does not only change the magnitude, but also the phase and frequency behavior of the measured impedance.

For the right, front, top region, the result during one breathing cycle is shown in Fig. 6a. Here, in order to show that the concept is valid for the complete frequency range, the current injection frequency was simulated to be $1 \mathrm{MHz}$. Again, the healthy reference data is shown in blue. In red, atelectasis was simulated inside the ROI (rft) and in green, the pathology was simulated outside the $\mathrm{ROI}$ in an adjacent lung lobe (rbt). In principle, the result for this ROI is similar to the result of the lbb region in Fig 5a A pathology inside the ROI has a higher impact on the measured impedance than a pathology in a different region. However, there are two differences between the $\mathrm{lbb}$ and $\mathrm{rtt}$ result: While in the lbb case, almost no ventilation is visible in the focused pathological case (red curve in Fig 5a), ventilation is still visible in the

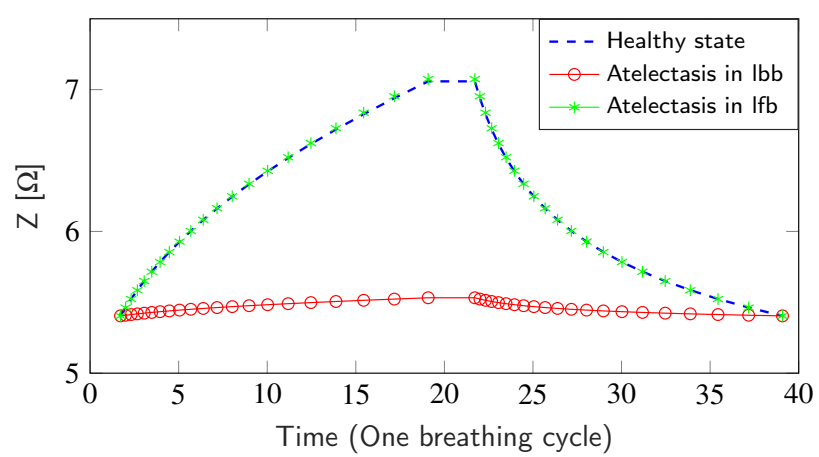

(a) Simulation result at $10 \mathrm{kHz}$ focused on the ROI lbb during one breathing cycle. The absolute value of the complex impedance is presented. In blue, all lung regions were in healthy state. In red, an atelectasis was simulated in the focused ROI lbb. In green, an atelectasis was simulated in the adjacent region Ifb.

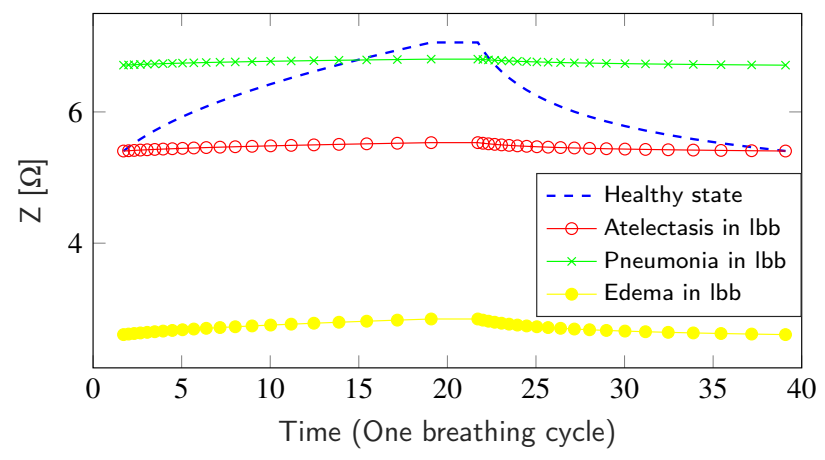

(b) Impact of the different simulated pathologies on the measurement result during a breathing cycle. Measurements were focused on the $\mathrm{lbb}$ region and simulated at a current frequency of $10 \mathrm{kHz}$. In blue, the healthy state is shown. Atelectasis is simulated in red, pneumonia in green and edema in yellow.

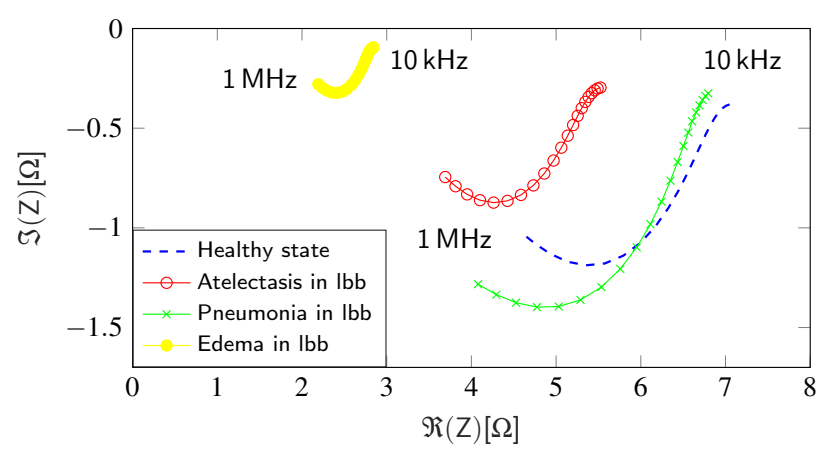

(c) Simulated impedance spectrum for different pathologies in the inhaled state from $10 \mathrm{kHz}$ to $1 \mathrm{MHz}$. The measurements are focused on the lbb region. In blue, the healthy state is shown. Atelectasis is simulated in red, pneumonia in green and edema in yellow.

Fig. 5: Simulation results for an impedance measurement focused on the left, back, bottom region of the lung. 


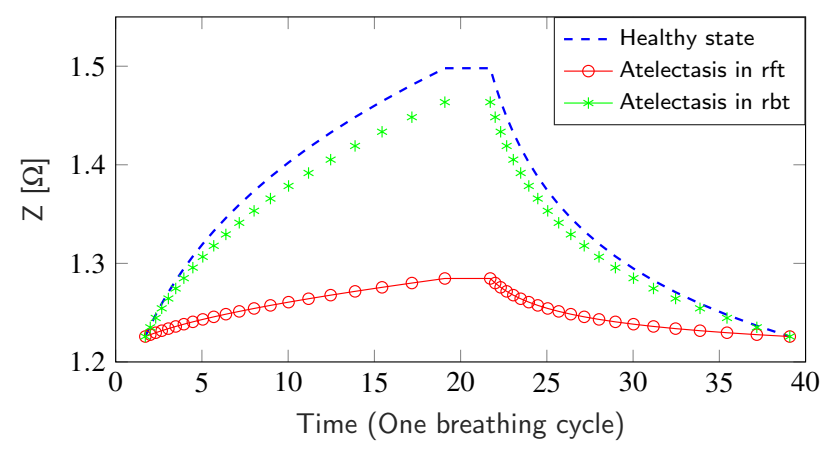

(a) Simulation result for an impedance measurement focused on the ROI rft during one breathing cycle. The absolute value of the complex impedance is presented. In blue, all lung regions were in healthy state. In red, an atelectasis was simulated in the focused ROI $\mathrm{rt}$. In green, atelectasis was simulated in the adjacent region rbt.

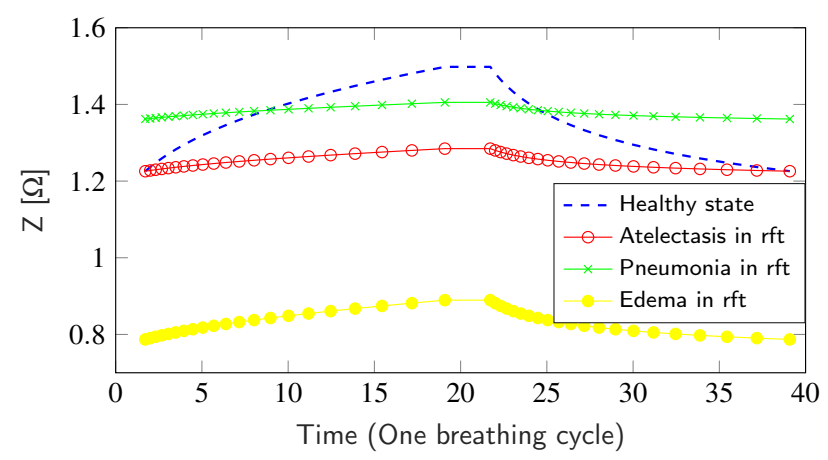

(b) Impact of the different simulated pathologies on the measurement result during a breathing cycle. Measurements were focused on the $\mathrm{rtt}$ region. In blue, the healthy state is shown. Atelectasis is simulated in red, pneumonia in green and edema in yellow.

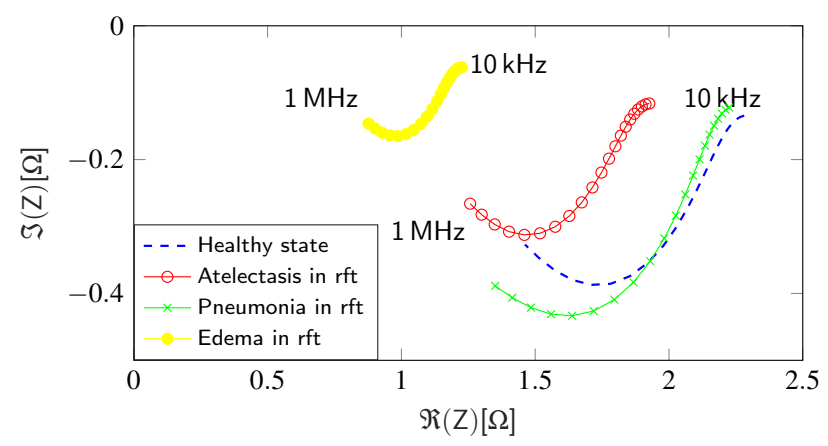

(c) Simulated impedance spectrum for different pathologies in the inhaled state. The measurements are focused on the $\mathrm{rft}$ region. In blue, the healthy state is shown. Atelectasis is simulated in red, pneumonia in green and edema in yellow.

Fig. 6: Simulation results for an impedance measurement focused on the right, front, top region of the lung. rft case (red curve in Fig 6a). Furthermore, the difference between the healthy state and the pathological state in a different region of the ROI is higher (difference between the blue and green curve in Fig $5 \mathrm{a}$ and Fig. 6a). This, however, is not surprising as the selectivity of the focusing configuration for the $\mathrm{rft}$ region is smaller than the selectivity for the Ibb region (9.15 vs. 31.3$)$. In the pathological state, the ventilation activity is limited to the non-pathological lung regions which are not focused by the electrode configuration. The lower the selectivity, the more of these changes outside the ROI will be represented in the impedance data. Thus, the temporal data shows the expected behavior.

Nevertheless, the lower selectivity configurations still amplify conductivity changes inside the ROI. This is demonstrated in Fig. 6b and Fig. 6c. In Fig. 6b, the impact of the different pathologies inside the ROI on the temporal data at $1 \mathrm{MHz}$ is shown. In Fig. 6c the respective impedance spectra are presented. Again, the impact of the pathology is clearly visible in both the temporal and the spectroscopic data.

\section{Comparison to EIT}

As mentioned in the Introduction, the general idea of EIT is to reconstruct conductivity changes inside the body from multiple impedance measurements at the body surface. Typically, this is done in a single plane. Thus, in order to compare the focused tetrapolar results to EIT, simulations were performed in the exact same model using a simulated electrode belt of 16 electrodes. For image reconstruction, the GREIT algorithm was used [13] (settings: number of targets $=500$, target-size $=0.03$, noise-figure $=0.5$ ). As EIT typically reconstructs conductivity changes with respect to a given reference, the inhaled state is referenced to the exhaled state.

In Fig. 7, the resulting EIT images for the healthy reference and the pathological scenarios are presented. In the top row, the pathology is present in the left, back, bottom region of the lung. The resulting image is similar for all three pathological cases. This is not surprising, as the pathological lobe does not contribute to the ventilation and thus does not change its conductivity between the exhaled and inhaled state. As EIT seeks to display conductivity changes, a time-invariant conductivity change inside a specific lobe does not have a high impact on the reconstructed images. Nevertheless, an analysis of the images allows the conclusion that ventilation activity is restricted in the back, left region. However, no three dimensional information can be extracted.

The images for a pathology in the right, front, top 

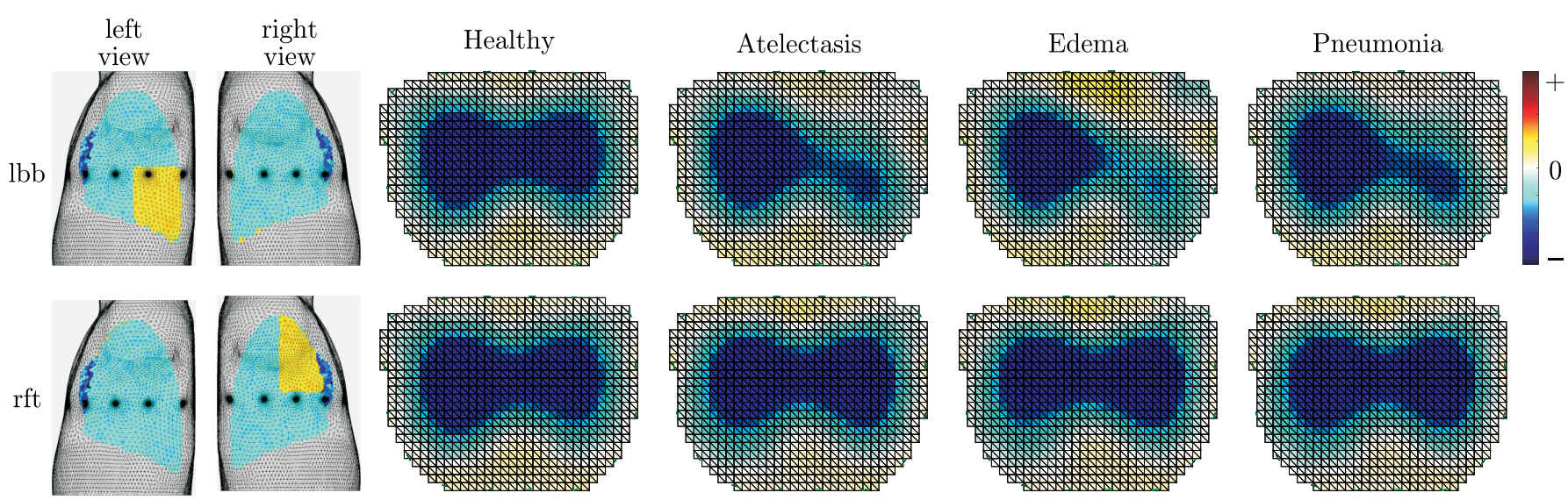

Fig. 7: Reconstructed EIT images based on simulated pathologies. In the top row, the healthy reference and pathological examples for the left, back, bottom region of interest are presented. In the bottom row, the same healthy reference and pathology for the right, front, top region of interest is shown. The respective region is highlighted in yellow in the left and right view. It can be seen that in the Ibb case, a high inhomogeneity can be observed whereas in the rft case, almost no impact on the EIT image is visible.

region are shown in the bottom row. Interestingly, a pathology inside this region has almost no impact on the reconstructed EIT images. The main reason for this is that the bottom regions of the lung have a higher volume than the top lung regions. This leads to a ventilation in the right front lung region, although only the bottom part of the front lung region is actually ventilated. A pure analysis of the EIT images would lead to the conclusion that the ventilation is homogeneously distributed, which is not the case in the pathological scenario.

In reality, of course, it is very unlikely that a specific lung region does not contribute to the ventilation while the other lobes are completely healthy. Nevertheless, some pathologies, such as lobar pneumonia, are primarily located in specific lobes. If misinterpretations due to reconstruction occur even in the simulated extreme case, there is a high possibility that EIT-image based criteria such as the ventilation inhomogeneity might over- or underestimate the real condition inside the lungs.

This result is not surprising, as EIT maps a three dimensional problem to one specific, two-dimensional image plane. The off plane sensitivity region of EIT is described to be a lens shaped region, which extends to approx. $\frac{1}{2}$ of the body diameter [24, 25]. The exact sensitivity region is, however, dependent on the geometry, the conductivity distribution inside the body, and the current injection and voltage measurement strategy. Furthermore, in single belt EIT, changes below and above the electrode plane can not be distinguished from each other. The proposed focused impedance measurement strategies overcomes this issue by addressing specific lung regions with individual electrode configurations. This, however, comes at the price of a decreased spatial resolution, as only regions can be analyzed, and a potentially higher effort in hardware, as four electrodes are needed for each region of interest.

\section{Experimental Results}

In order to validate the simulation results in an experimental setup, a water tank experiment was performed. The Draeger EEK2 EIT device was attached to the water tank phantom as described in the Materials and Methods section. To remove noise, the data was filtered with a simple mean-median filter. Electrodes were placed both in a single plane for EIT measurements and in configurations focusing the bottom left and top right region. In Fig. 8, the result of the experimental study is shown. The measurements are split into five phases. In phase I, all balloons were ventilated. The EIT image shows a typical result with an impedance change in both the left and right lung. Also, the raw data of the configurations focused on the bottom left and top right region shows normal ventilation.

At $30 \mathrm{~s}$, the valve to the bottom left lobe was closed so that on the left side ventilation was only present in the top region while the right side was homogeneously ventilated in both the bottom and top region (phase II). In the EIT image, a decrease in impedance change in the left lung can be observed. In the raw data of the focused impedance measurement, almost no ventilation activity can be seen in the left bottom focusing configuration. In contrast, the right top focusing configuration shows a similar behavior as during the baseline measurement.

In phase III, the top left region is disabled while the other regions are normally ventilated. Similar to phase II, the impedance change in the EIT image decreases compared to the baseline. When comparing phase II and 
I: Baseline II: left bottom III: left top
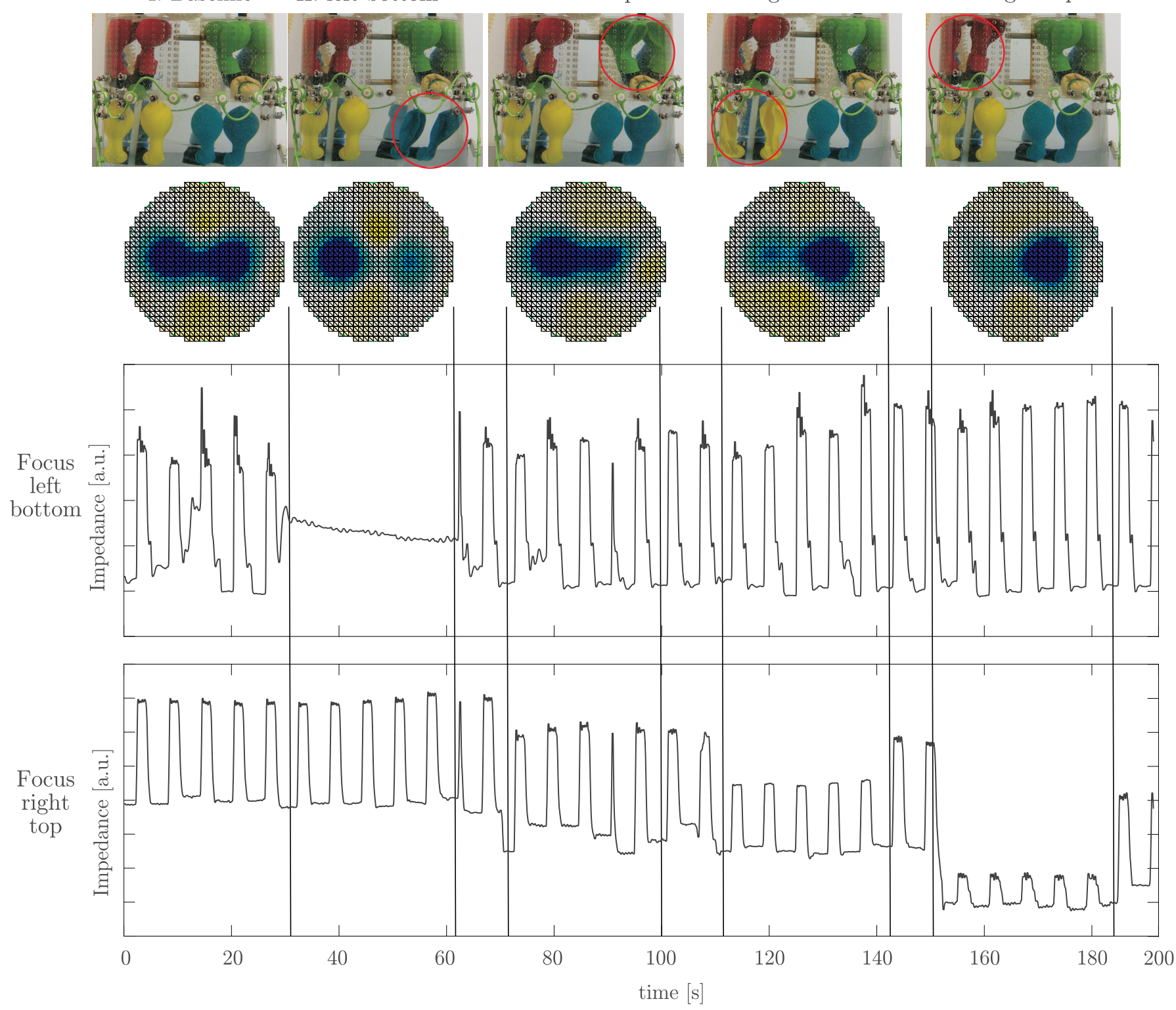

Fig. 8: Result of the experimental validation at the water phantom in five phases $(I-V)$. At each phase, the balloons, which represent specific lung lobes, were disabled in the respective region as highlighted in the second row. The corresponding EIT image is shown for each phase. The images show a difference to the baseline measurement. However, no information on the vertical location of the disabled region can be extracted. Below the images, the filtered raw data of two focused electrode configurations (left bottom and right top) is shown. The location of the disabled balloons can be clearly seen in the respective focused impedance data. 
phase III to each other, the EIT image shows a shift in impedance activity towards the center. In reality, however, no ventilation is present in the center but the ventilation occurs in distinct regions on the left and right side. In the raw data, the left bottom configuration shows a similar signal as in the baseline measurement. In the right top region, however, a small shift in the base impedance can be observed, while the amplitude of the impedance change remains constant.

In phase IV, the right bottom region is disabled. In the EIT image, the expected result is visible as the impedance change in the right lung appears to be decreased. In the raw data, again, almost no impact on the signal of the configuration focusing the left bottom region can be seen. In the signal in the right top configuration, however, a change in both baseline and ventilation amplitude is visible.

Finally, in phase $\mathrm{V}$, the top right region is disabled. The EIT image is very similar to the image in phase IV, so that a decrease in impedance activity is present in the right lung region. When observing the raw data, the left bottom focusing configuration is not changed by the lack of ventilation in the right top region. The bottom right configuration, however, shows a big signal change. Both the baseline and the change in amplitude due to ventilation are decreased significantly. Nevertheless, ventilation activity is still visible in this data even though no ventilation is present in the focus region.

Overall, the experimental results confirm the simulations. In EIT, a general decrease in ventilation inside the left and right lung can be detected. However, it is unclear at which height the lack of ventilation exists. Furthermore, it cannot be distinguished, if the ventilation is decreased in the top and bottom region by $50 \%$ or if one region is completely collapsed. In contrast, in the raw data, the affected region can be identified. In the experiment the data focused on the left bottom region showed a much better result than the configuration focused on the right top region. This, however, can be explained by the physical realization of the water tank: First, the electrode positions are fixed and cannot be adjusted. Thus, it was not possible to place the electrodes optimally, especially in the right top region. Nevertheless, a high selectivity was achieved for the left bottom region while the right top region apparently has a lower selectivity, so that impedance changes in other regions have an impact on the measured data as well. Second, the inflation and deflation of the balloons causes the water level to change as well. This has a higher impact on the top regions than on the bottom regions. In total, however, even in the less focusing right top configuration the non-ventilated case is clearly visible.

Even more, by combination of multiple measurements (for example top and bottom right), a more precise localization should be possible.

\section{Discussion and Conclusion}

Our results indicate that focused impedance measurements can be a useful tool to provide a three-dimensional, pulmonary monitoring of desired regions of interest. In simulations, it was possible to focus impedance measurements to specific regions of the lung using single tetrapolar measurements. Activity from different, adjacent lung regions outside the focus region had almost no effect on the measured data. Furthermore, a spectroscopic analysis of the data in one specific time point revealed that each pathology has a different impact on the impedance spectrum. Thus, the method could not only be used to detect the presence of a pathology inside a specific region by temporal analysis, but also could help in identifying the kind of pathology which is present inside the ROI. In a water tank experiment, the focusing ability of specific electrode configurations was verified in measured data. When comparing the presented method to EIT, it was demonstrated that EIT has the risk of overor underestimating impedance changes above or below the image plane. Nevertheless, EIT still has multiple advantages over the presented method such as the higher spatial resolution and the easy interpretation of data. Furthermore, the application of a single electrode belt is easier to realize in clinical practice than the attachment of individual electrodes for each individual region of interest.

Several limitations and restrictions apply to the study. First of all, the problem and the model were simplified in various ways. The background of the body model was modeled as muscle tissue. No noise or disturbances were added in the simulations. Furthermore, pathologies were modeled to cause a complete lung failure in a specific lung region while the remaining regions were healthy. In reality, of course, even a local pathology does not provide this sharp spatial transition from pathological to healthy tissue. Second, simulations were only performed for eight regions of interest. Additionally, only single tetrapolar measurements were analyzed for each ROI. While this gives a good overview about the principal effects, a higher spatial resolution would be desirable for a potential clinical use. This could be achieved by either adding more configurations focusing smaller regions of interest, or by combining multiple measurements. Furthermore, a visualization strategy needs to be implemented. One of the biggest advantage of EIT is the easy interpretation of 
the images. In our approach, the raw data is analyzed, which is hard to interpret in a real time scenario. One possibility would be a model based visualization, where the respective lung regions are animated according to the measured data allowing an easy access to the provided information. Finally, all results were obtained in simulations or the water tank phantom and need to be validated in-vivo.

Nevertheless, the presented study provides an insight into the concept of focused bioimpedance measurements. These might not only be interesting in the field of pulmonary monitoring, but can be applied to other fields, where a local analysis of specific regions of interest is desirable, such as cardiac monitoring.

\section{Acknowledgment}

The authors gratefully acknowledge financial support provided by the German Research Foundation [Deutsche Forschungsgemeinschaft (DFG), LE 817/20-3, CZ 215/2-3].

\section{Conflict of interest}

Authors state no conflict of interest.

\section{References}

1. Thompson BT, Chambers RC, Liu KD. Acute Respiratory Distress Syndrome. New England Journal of Medicine. 2017 aug;377(6):562-572. http://dx.doi.org/10.1056/nejmra1608077

2. Blankman P, Hasan D, Erik G, Gommers D. Detection of 'best' positive end-expiratory pressure derived from electrical impedance tomography parameters during a decremental positive end-expiratory pressure trial. Crit Care. 2014;18(3):R95. http://dx.doi.org/10.1186/cc13866

3. Zhao Z, Pulletz S, Frerichs I, Müller-Lisse U, Möller K. The EIT-based global inhomogeneity index is highly correlated with regional lung opening in patients with acute respiratory distress syndrome. BMC Research Notes. 2014;7(1):82. http://dx.doi.org/10.1186/1756-0500-7-82.

4. Trepte CJC, Phillips CR, Solà J, Adler A, Haas SA, Rapin $M$, et al. Electrical impedance tomography (EIT) for quantification of pulmonary edema in acute lung injury. Critical Care. $2015 \mathrm{dec} ; 20(1)$. http://dx.doi.org/10.1186/s13054-015-1173-5

5. Leonhardt S, Lachmann B. Electrical impedance tomography: the holy grail of ventilation and perfusion monitoring? Intensive Care Med. 2012

Dec;38(12):1917-1929. http://dx.doi.org/10.1007/s00134-012-2684-z
6. Orschulik J, Petkau R, Wartzek T, Hochhausen N, Czaplik M, Leonhardt S, et al. Improved electrode positions for local impedance measurements in the lung-a simulation study. Physiological Measurement. 2016 nov;37(12):2111-2129. http://dx.doi.org/10.1088/0967-3334/37/12/2111

7. Orschulik J, Hochhausen N, Czaplik M, Teichmann D, Leonhardt S, Walter M. Addition of internal electrodes is beneficial for focused bioimpedance measurements in the lung. Physiological Measurement. 2018 feb;39(3):035009. http://dx.doi.org/10.1088/1361-6579/aaad45.

8. Graham BM, Adler A. Electrode placement configurations for 3D EIT. Physiological Measurement. 2007 jun;28(7):S29-S44. http://dx.doi.org/10.1088/0967-3334/28/7/s03.

9. Borsic A, Halter R, Wan Y, Hartov A, Paulsen KD. Sensitivity study and optimization of a 3D electric impedance tomography prostate probe. Physiological Measurement. 2009 jun;30(6):S1-S18. http://dx.doi.org/10.1088/0967-3334/30/6/s01.

10. Wagenaar J, Adler A. Electrical impedance tomography in 3D using two electrode planes: characterization and evaluation. Physiological Measurement. 2016 may;37(6):922-937. http://dx.doi.org/10.1088/0967-3334/37/6/922

11. Schullcke B, Krueger-Ziolek S, Gong B, Mueller-Lisse U, Moeller K. Simultaneous application of two independent EIT devices for real-time multi-plane imaging. Physiological Measurement. 2016 aug;37(9):1541-1555. http://dx.doi.org/10.1088/0967-3334/37/9/1541

12. Martin S, Choi CTM. A Post-Processing Method for Three-Dimensional Electrical Impedance Tomography. Scientific Reports. 2017 aug;7(1). http://dx.doi.org/10.1038/s41598-017-07727-2

13. Adler A, Arnold JH, Bayford R, Borsic A, Brown B, Dixon $P$, et al. GREIT: a unified approach to $2 \mathrm{D}$ linear EIT reconstruction of lung images. Physiological Measurement. 2009 Jun;30(6):S35-S55. http://dx.doi.org/10.1088/0967-3334/30/6/s03.

14. Grychtol B, Müller B, Adler A. 3D EIT image reconstruction with GREIT. Physiological Measurement. 2016 may;37(6):785-800. http://dx.doi.org/10.1088/0967-3334/37/6/785

15. Schullcke B, Gong B, Krueger-Ziolek S, Tawhai M, Adler $A$, Mueller-Lisse $U$, et al. Lobe based image reconstruction in Electrical Impedance Tomography. Medical Physics. 2017 jan;44(2):426-436. http://dx.doi.org/10.1002/mp.12038.

16. RABBANI KS, SARKER M, AKOND MHR, AKTER T. Focused Impedance Measurement (FIM): A New Technique with Improved Zone Localization. Annals of the New York Academy of Sciences. 1999 apr;873(1 
ELECTRICAL BI):408-420.

http://dx.doi.org/10.1111/j.1749-6632.1999.tb09490.x

17. Geselowitz DB. An application of electrocardiographic lead theory to impedance plethysmography. IEEE Trans Biomed Eng. 1971 Jan;18(1):38-41.

18. Puikkonen JJ, Malmivuo J. Theoretical Investigation of the Sensitivity Distribution of Point EEG Electrodes on the Three Concentric Spheres Model of a Human Head: An Application of the Reciprocity Theorem. Tampere University of Technology; 1987.

19. Väisänen O, Malmivuo J. Improving the SNR of EEG generated by deep sources with weighted multielectrode leads. Journal of Physiology-Paris. 2009 nov; 103(6):306-314. http://dx.doi.org/10.1016/j.jphysparis.2009.07.003

20. Adler A, Lionheart WRB. Uses and abuses of EIDORS: an extensible software base for EIT. Physiological Measurement. 2006 apr;27(5):S25-S42. http://dx.doi.org/10.1088/0967-3334/27/5/s03.

21. Schöberl J. NETGEN An advancing front 2D/3D-mesh generator based on abstract rules. Computing and Visualization in Science. 1997 jul;1(1):41-52. http://dx.doi.org/10.1007/s007910050004

22. Gabriel S, Lau RW, Gabriel C. The dielectric properties of biological tissues: III. Parametric models for the dielectric spectrum of tissues. Phys Med Biol. 1996

Nov;41(11):2271-2293. http://dx.doi.org/10.1088/0031-9155/41/11/003

23. American Thoracic Society, Infectious Diseases Society of America. Guidelines for the Management of Adults with Hospital-acquired, Ventilator-associated, and Healthcare-associated Pneumonia. American Journal of Respiratory and Critical Care Medicine. 2005

feb;171(4):388-416. http://dx.doi.org/10.1164/rccm.200405-644ST.

24. Putensen C, Zinserling J, Wrigge H. Electrical Impedance Tomography for Monitoring of Regional Ventilation in Critically III Patients. In: Vincent JL, editor. Intensive Care Medicine. New York, NY: Springer New York; 2006. p. 448-457. http://dx.doi.org/10.1007/0-387-35096-9_41

25. Adler A, Frerichs I, Grychtol B. The off-plane sensitivity of EIT. Proc EIT (Neuchâtel, Switzerland,). 2015;p. 68. 